\title{
Assessing alcohol consumption through wastewater-based epidemiology: Spain as case study
}

\author{
Ester López-García ${ }^{1}$, Carlos Pérez López ${ }^{1}$, Cristina Postigo ${ }^{1 *}$, Vicente Andreu ${ }^{2}$, Lubertus \\ Bijlsma $^{3}$, Iria González-Mariño ${ }^{4,5}$, Félix Hernández ${ }^{3}$, Rosa Maria Marcé6 ${ }^{\text {, Rosa Montes }}{ }^{4}$, \\ Yolanda Picó ${ }^{2}$, Eva Pocurull ${ }^{6}$, Andreu Rico ${ }^{7}$, Rosario Rodil ${ }^{4}$, María Rosende ${ }^{8}$, Yolanda \\ Valcárcel $^{9}$, Olatz Zuloaga ${ }^{10}$, José Benito Quintana ${ }^{4}$, Miren López de Alda ${ }^{1 *}$
}

\footnotetext{
${ }^{1}$ Water, Environmental, and Food Chemistry Unit (ENFOCHEM), Department of Environmental Chemistry, Institute of Environmental Assessment and Water Research (IDAEA-CSIC), Barcelona

${ }^{2}$ Food and Environmental Safety Research Group (SAMA-UV), Desertification Research Centre (CIDE), CSIC-Generalitat Valenciana-University of Valencia, Valencia

${ }^{3}$ Environmental and Public Helath Analytucal Chemistry, Research Institute for Pesticides and Water, University Jaume I, Castellón

${ }^{4}$ Department of Analytical Chemistry, Nutrition and Food Sciences, Institute of Research on Chemical and Biological Analysis (IAQBUS), Universidade de Santiago de Compostela, Santiago de Compostela

${ }^{5}$ Department of Analytical Chemistry, Nutrition and Bromatology, Faculty of Chemical Sciences, University of Salamanca, Salamanca.

${ }^{6}$ Department of Analytical Chemistry and Organic Chemistry, Universitat Rovira i Virgili, Tarragona

${ }^{7}$ IMDEA Water Institute, Science and Technology Campus of the University of Alcalá, Alcalá de Henares

${ }^{8}$ FI-TRACE group, Department of Chemistry, University of the Balearic Islands, Palma de Mallorca ${ }^{9}$ Research Group in Environmental Toxicology and Risk Assessment (TAyER), Medical specialities and Public Helath, Faculty of Health Sciences, Rey Juan Carlos University, Madrid

${ }^{10}$ Department of Analytical Chemistry, Faculty of Science and Technology (UPV/EHU) \& Plentzia Marine Station of Basque Country University (EHU/UPV), Basque Country
}

*Corresponding Authors: Cristina Postigo; Miren López de Alda Institute of Environmental Assessment and Water Research (IDAEA-CSIC) Department of Environmental Chemistry C/ Jordi Girona 18-26, 08034 Barcelona, Spain. cprqam@cid.csic.es; miren.lopezdealda@idaea.csic.es Tel: +34-934-006-100, Fax: +34-932-045-904 


\section{Abstract}

Background: Estimating alcohol consumption in the population has received great interest given the social, health and economic problems that it generates. In this study, wastewaterbased epidemiology (WBE), an alternative method to estimate licit and illicit drug consumption rates in a given population through the analysis of chemicals and/or metabolites in wastewaters, was applied to estimate alcohol consumption at the local and national level in Spain.

Methods: Composite (24-h) wastewater samples were collected at the inlet of 17 wastewater treatment plants (WWTPs) located in 13 cities for seven consecutive days in spring of 2018. The sampled area covered $12.8 \%$ of the Spanish population. Wastewater samples were analyzed by an ion-pair liquid chromatography-tandem mass spectrometry (LC-MS/MS) method to determine the concentration of ethyl sulphate (EtS), the biomarker used to back-calculate alcohol consumption.

Results: Alcohol consumption ranged from 4.5 to $46 \mathrm{~mL} /$ day/inhabitant. Differences in consumption were statistically significant among the investigated cities and also between weekdays and weekends. At the local level, in each investigated population WBE provided, mostly, estimates of alcohol consumption comparable to those reported by its corresponding region in the Spanish National Health Survey. Also at the national level, comparable results were obtained between WBE-derived annual consumption rate $(5.7 \pm 1.2 \mathrm{~L}$ ethanol per capita (aged 15+) and that reported for Spain in the National Health Survey (4.7 L ethanol per capita (aged 15+)).

Conclusions: This is the largest WBE study carried out to date in Spain to estimate alcohol consumption rates. It confirms that this approach is useful for establishing spatial and temporal patterns of alcohol consumption which could contribute to the development of health care management plans and policies. Nevertheless, further studies are needed to reduce the uncertainties associated with WBE and to obtain more comparable data with established indicators.

1




\section{Introduction}

In 2016, the consumption of alcohol was responsible for 3 million deaths worldwide and it became one of the main health risk factors for the population, being more harmful than digestive diseases, road injuries, diabetes or violence (World Health Organization (WHO), 2018). In Spain, alcohol is the psychoactive substance most consumed (Observatorio Español de las Drogas y las Adicciones (OEDA), 2019). In 2017 (last reported year), 91\% of the Spanish population aged 15-64 years had consumed alcohol at some point in their lifetime, while 75\% had consumed alcohol in the last year, and $63 \%$ did it in the last month. Overall, the consumption by men is higher than by women and the average age at which alcohol begins to be consumed is 16.6 years (OEDA, 2019). According to the 2018's Global status report on alcohol and health provided by the WHO, the annual intake of alcohol in Spain in 2016 was 10 L of pure alcohol per capita (aged 15+), which is similar to the European average (9.8 L) (WHO, 2018). These estimates are traditionally obtained from population surveys, recorded alcohol data (alcohol taxation or sales) and unrecorded alcohol data (homemade or informally produced alcohol, smuggled alcohol, alcohol for industrial or medical uses, alcohol obtained through cross-border shopping, or surrogate alcohol) (WHO, 2018). Through surveys, consumption figures can be disaggregated for specific population groups by age or gender. However, the use of these tools/data to derive alcohol consumption figures is time consuming and relatively expensive, and consequently it does not allow obtaining real-time estimates (i.e., consumption data in Spain are given with a delay of two years). Furthermore, the data obtained by surveys may not be representative of actual population consumption due to misreport of alcohol consumption by survey participants (Stockwell et al., 2016; van Wel et al., 2016) or to inaccurate estimates of unrecorded alcohol (Probst et al., 2019). Therefore, it is necessary to propose alternative approaches that provide quick and precise information and that, together with the traditional ones, can help to obtain a more reliable picture of alcohol consumption rates.

Wastewater-based epidemiology (WBE) is a novel approach that has been applied in the last decade to estimate illicit drug use at city level (González-Mariño et al., 2019). The European Monitoring Centre for Drugs and Drug Addiction (EMCDDA) has adopted it, indeed, as a complementary indicator to established methods for illicit drugs use estimation (EMCDDA, 2016). The WBE approach is based on the fact that, after consumption, the substances are excreted via urine and faeces, either unaltered or as a metabolite, and conducted through the sewage network to a wastewater treatment plant (WWTP). Thus, a raw wastewater sample contains specific biomarkers of the drugs that can be used to back-calculate the amount of substance that has been actually consumed. In the case of alcohol, after human consumption, about $95 \%$ is metabolized in the liver via oxidation to acetaldehyde and acetic acid, about $5 \%$ is excreted unaltered, and a small part $(<0.1 \%)$ is excreted as ethyl sulphate (EtS) and ethyl glucuronide $(\mathrm{EtG})$ after conjugation with sulphate and glucuronic acid, respectively. EtS and EtG can be detected in urine after 1 hour of alcohol intake (Helander and Beck, 2005), so they have been proposed as good indicators for recent alcohol consumption. However, only EtS is stable in wastewater (Rodríguez-Álvarez et al., 2014) and its occurrence in wastewater is exclusively due to alcohol consumption and not to the metabolism of unaltered alcohol by 
endogenous bacteria (Reid et al., 2011). Thus, EtS has been pointed out as the best biomarker to estimate alcohol consumption by means of WBE.

WBE was first applied to estimate alcohol consumption in 2011 in Oslo (Norway) (Reid et al., 2011) and, since then, many studies have been carried out in cities from other European countries (Andrés-Costa et al., 2016; Baz-Lomba et al., 2016; Gatidou et al., 2016; Mastroianni et al., 2014, 2017; Rodríguez-Álvarez et al., 2014, 2015; van Wel et al., 2016) Vietnam (Nguyen et al., 2018), China (Gao et al., 2020), United States (Chen et al., 2019), Canada (Ryu et al., 2016), and Australia (Zheng et al., 2020). The main objective of these studies was not only to investigate spatial differences of alcohol consumption between populations or to assess changes in alcohol consumption due to special events (Andrés-Costa et al., 2016), but also, to compare WBE-derived alcohol estimates with alcohol consumption figures obtained by means of traditional methods, such as, official data provided by the WHO or by national surveying institutions. In these studies, the alcohol consumption rates were estimated from data gathered from a single WWTP, which only serves a city or part of it, after a sampling period of one week in most of the cases, except for Milan and Santiago (Rodríguez-Álvarez et al., 2015), Oslo (Reid et al., 2011), Lied (Belgium) (van Wel et al., 2016), U.S (Chen et al., 2019) and Australia (Zheng et al., 2020), for which longer sampling periods were used (namely, 2 weeks, 3 weeks, four-two weeks periods, one weekday every month during eleven months, and one week every two months during 6 years, respectively). To date, only two studies have conducted nation-wide investigations by collecting samples from different WWTPs: a study conducted in Australia, in which 18 WWTPs were sampled, covering $45 \%$ of the whole population (Lai et al., 2018); and another one carried out in Belgium, which covered 8 WWTPs and 12.8\% of the total population (Boogaerts et al., 2016).

The present study is one of the few nation-wide applications of WBE to estimate alcohol consumption rates, and the largest conducted so far in Spain. Wastewater samples were analyzed from 17 WWTPs, covering $12.8 \%$ of the Spanish population. The specific objectives of this work were: i) to assess spatial differences in alcohol consumption between the different investigated areas in Spain, ii) to assess weekly consumption patterns, and iii) to extrapolate the estimated alcohol consumption in the studied areas to the whole Spanish population, and to compare it with official data reported by the WHO or national institutions.

\section{Material and methods}

\subsection{Reagents}

Analytical standards of ethyl sulphate (EtS) and its isotopically labeled compound, EtS- $\mathrm{d}_{5}$, were obtained as EtS sodium salt and ethyl-d $\mathrm{d}_{5}$ sulphate salt from Cerilliant (Round Rock, TX, USA) as solutions in methanol $(\mathrm{MeOH})$ at a concentration of $1 \mathrm{mg} / \mathrm{mL}$. Water and $\mathrm{MeOH}$, both HPLC grade, and acetic acid (98\% purity) used as a mobile phase modifier, were purchased from Merck (Darmstadt, Germany). Dibutylamine ( $>99.5 \%$ purity), also used as a mobile phase modifier, was obtained from Sigma Aldrich (Steinheim, Germany). 


\subsection{Standard solutions}

Stock standard solutions were prepared at different concentrations in the range of 10 to 20,000 $\mu \mathrm{g} / \mathrm{L}$ by appropriate dilution of the commercial EtS standard in $\mathrm{MeOH}$, with a constant concentration of EtS- $\mathrm{d}_{5}$ of $2,500 \mu \mathrm{g} / \mathrm{L}$, and were stored in the dark at $-20^{\circ} \mathrm{C}$ until analysis. Before analysis, working standard solutions were freshly prepared by dilution of these stock standard solutions in HPLC water $(1: 100, \mathrm{v} / \mathrm{v})$.

\subsection{Sample collection and preparation}

Influent wastewater samples were collected from 17 WWTPs located in 13 Spanish cities that belong to 7 out of the 17 regions of Spain. Figure S1 in the Supporting Information shows the location of the sampled WWTPs. The sampling covers populations of various sizes (i.e, between 47,961 and 1,163,154 inhabitants). In total, the population reached with the sampling was $5,981,848$ inhabitants, which corresponds to $12.8 \%$ of the Spanish population. The cities sampled were Barcelona, Bilbao, Castellón, Guadalajara, Lleida, Madrid, Móstoles, Palma de Mallorca, Reus, Santiago de Compostela, Tarragona, Toledo, and Valencia, including in some cases part of their metropolitan area. Except for Barcelona, Madrid and Móstoles, where WWTPs only covered 35, 30 and $90 \%$ of their total population, respectively, all other main cities were fully covered (100\% of their population). Table S1 shows the populations served by each WWTP as well as the sampling protocol carried out in each of them.

From each WWTP, 24-h composite influent wastewater samples were collected during seven consecutive days in spring of 2018 using time or flow proportional techniques (Table S1). The sampling was conducted during a "normal week", so that special events such as holidays or festivals were avoided. After collection, samples were immediately stored at $-20^{\circ} \mathrm{C}$. They were sent frozen by courier in less than 24 hours to the laboratory in Barcelona, where all samples were analyzed. Once in the laboratory, an aliquot of $10 \mathrm{~mL}$ was spiked with EtS- $\mathrm{d}_{5}$ at a concentration of $25 \mu \mathrm{g} / \mathrm{L}$ and one $\mathrm{mL}$ of this sample was transferred to a $1.5 \mathrm{~mL}$ microcentrifuge tube and centrifuged at 10,000 rpm for 10 minutes at a temperature of $4^{\circ} \mathrm{C}$ (Eppendorf 5810R, Hamburg, Germany). Then, the supernatant was transferred to a glass vial and stored at $-20^{\circ} \mathrm{C}$ in the darkness until its analysis by liquid chromatography coupled to tandem mass spectrometry (LC-MS/MS).

\subsection{Sample analysis}

The analysis of EtS was performed with a previously described methodology based on ion-pair LC-MS/MS (Mastroianni et al., 2014) using a Symbiosis ${ }^{\mathrm{TM}}$ Pico System (Spark Holland, Emmen, The Netherlands) equipped with a $100 \mu \mathrm{L}$ sample loop. The LC system was coupled to a 4000QTRAP hybrid triple quadrupole-linear ion trap (QqLIT) mass spectrometer equipped with a Turbo Ion Spray source (AB-Sciex, Foster City, CA, USA) set in the negative ionization mode (ESI-). Chromatographic separation was performed with a Purospher Star RP-18 endcapped column $(125 \mathrm{~mm} \times 2 \mathrm{~mm}$, particle size $5 \mu \mathrm{m})$ preceded by a guard column of the same packing material and particle size, both from Merck (Darmstad, Germany) and a mobile phase consisting of $\mathrm{MeOH}$ and water both containing $5 \mathrm{mM}$ of dibutylammonium acetate (DBAA) at a constant flow rate of $0.3 \mathrm{~mL} / \mathrm{min}$. MS/MS detection was performed in selected reaction 
monitoring mode $(\mathrm{SRM})$ recording $2 \mathrm{SRM}$ transitions for $\mathrm{EtS}(125 \rightarrow 97,125 \rightarrow 80)$ and one for EtS-d $5(130 \rightarrow 98)$. Data acquisition and evaluation was performed with Analyst 1.5 software (AB-Sciex, Foster City, CA, USA). Quantification of the samples was based on the isotope dilution method.

\subsection{Alcohol consumption estimates}

Back calculation of alcohol consumption was made according to the following equation:

$$
\frac{m L \text { EtOH }}{\text { day } * \text { inhabitant }}=C_{E t S}\left[\frac{\mu g}{L}\right] * 10^{-6}\left[\frac{g}{\mu g}\right] * Q\left[\frac{m^{3}}{\text { day }}\right] * 10^{3}\left[\frac{L}{m^{3}}\right] * \frac{1}{P} * 3047 * \frac{1}{\rho_{\text {EtoH }\left(\frac{g}{m L}\right)}}
$$

where $\mathrm{C}_{\mathrm{EtS}}$ is the concentration of EtS measured in the wastewater sample, $\mathrm{Q}$ is the water flow entering the WWTP, P is the total population served by the WWTP (Table S1), 3047 is the correction factor applied which takes into account the molar mass ratio between ethanol (MW: $46.07 \mathrm{~g} / \mathrm{mol}$ ) and EtS (MW: $126.13 \mathrm{~g} / \mathrm{mol}$ ) and the excretion rate of EtS in urine $(0.012 \%)$ (Rodríguez-Álvarez et al., 2015), and $\rho_{\mathrm{EtOH}}$ is ethanol density $(0.789 \mathrm{~g} / \mathrm{mL}$ ).

\subsection{Statistical data analysis}

Data were statistically analysed to compare alcohol consumption rate between populations, regions, weekdays and weekend, and between populations grouped according to their size (above or below 300,000 inhabitants). Since data were not normally distributed (after Shapiro Wilk test) or sample size was too small $(\mathrm{n}<10)$ in some cases, non-parametric tests were applied. The U Mann Whitney test was used to compare two independent samples, whereas Kruskal Wallis test was used to compare three or more individual groups. If the latter revealed significant differences among groups, they were subsequently investigated after applying $U$ Mann Whitney test to each two populations. False Discovery Rate (FDR) correction for multiple testing was applied to reduce the number of "false positive". Spearman correlation test was also applied to assess correlation between WBE-derived data and those reported by established indicators. All the analysis were done using the software R (version R 3.5.3) and considering a $95 \%$ confidence level $(\alpha=0.05)$.

\section{Results}

\subsection{Occurrence of EtS in wastewater samples and alcohol consumption estimations}

Table 1 shows the concentrations of EtS, the mass loads of EtS that reached each WWTP and the estimated alcohol consumption in each investigated area, expressed as average, median and range; whereas Figure 1 depicts alcohol consumption in the form of boxplots by each investigated population and in the various considered regions. EtS was found in all samples above LOQ $(0.07 \mu \mathrm{g} / \mathrm{L})$ at concentrations ranging from $1.4 \mu \mathrm{g} / \mathrm{L}$ (Santiago de Compostela) to $74 \mu \mathrm{g} / \mathrm{L}$ (Tarragona). The average weekly concentrations of EtS ranged from 2.9 to $43 \mu \mathrm{g} / \mathrm{L}$, with the lowest values being found in the WWTPs that serve the cities of Santiago de Compostela, Lleida, and Guadalajara (below $10 \mu \mathrm{g} / \mathrm{L}$ ) and the highest values in the WWTPs 
that serve Móstoles $(31 \mu \mathrm{g} / \mathrm{L})$ and Tarragona $(43 \mu \mathrm{g} / \mathrm{L})$. The average weekly levels of EtS measured in the remaining WWTPs were between 11 (Toledo) and $21 \mu \mathrm{g} / \mathrm{L}$ (Reus).

The alcohol consumption estimated from levels of EtS in the analyzed samples ranged from 4.5 (Santiago de Compostela) to $46 \mathrm{~mL} /$ day/inhabitant (Tarragona). The cities with the highest average alcohol consumption were Tarragona, Bilbao, and Móstoles, with average weekly consumption of 27,20 , and $17 \mathrm{~mL} /$ day/inhabitant, respectively. The lowest average alcohol consumptions ( $<10 \mathrm{~mL} /$ day/inhabitant) were estimated in Toledo (7.4), Santiago de Compostela (8.4), Lleida (8.5), Madrid-Centre (8.9), Castellón (9.0), and Valencia-QB (9.4). In the remaining investigated areas (Guadalajara, Barcelona, Reus, Madrid-North, Valencia-PI, Valencia-PII and Palma de Mallorca), average alcohol consumption was between 11 and 14 mL/day/inhabitant.

Comparing with previous studies conducted in Spain, similar alcohol consumption rates were previously reported in Barcelona (18 mL/day/inhabitant (aged 15+)) (Mastroianni et al., 2017) and Castellón (6.6 mL/day /inhabitant) (Baz-Lomba et al., 2016). On the contrary, higher alcohol consumption in Santiago de Compostela (13.6-16.3 mL/day/inhabitant) (RodríguezÁlvarez et al., 2015, 2014), and lower alcohol consumption in Valencia-PI (6.2 $\mathrm{mL} /$ day/inhabitant (aged 15+)), Valencia-PII (3 mL/day/inhabitant (aged 15+)) and ValenciaQB (9.4 mL/day/inhabitant (aged 15+)) (Andrés-Costa et al., 2016) were previously reported.

Comparing with other international studies, the estimated rates in the investigated Spanish populations (average alcohol consumption from 7.4 to $27 \mathrm{~mL} /$ day/inhabitant), were similar to those reported by other investigated cities (Table S2) except in Ho Chin Minh (Vietnam) (Nguyen et al., 2018), Lesvos (Greece) (Gatidou et al., 2016), Milan (Italy) (Baz-Lomba et al., 2016; Rodríguez-Álvarez et al., 2015) and Lugano (Switzerland) (Ryu et al., 2016) where alcohol consumption rates (from 3.4 to $6.6 \mathrm{~mL}$ /day/inhabitant) were lower than those estimated for Spanish populations. On the contrary, Copenhagen (Denmark) and Granby (Canada) (Ryu et al., 2016), showed higher alcohol consumption rates, 40 and $44 \mathrm{~mL} /$ day/inhabitant, respectively.

\subsection{Spatial variation in alcohol consumption}

Statistical test applied to evaluate spatial variation in alcohol consumption among different population showed that populations belonging to the same region showed no statistically significant differences in alcohol consumption ( $\mathrm{p}$-value > 0.05, U Mann Whitney test) (Table S3) while, statistically significant differences between populations belonging to different regions were found ( $\mathrm{p}$-value $<0.05$, U Mann Whitney test) (Table S3). Particularly, alcohol consumption estimated for the population served by Bilbao WWTP was different to that observed in 9 other populations, namely, Castellón, Guadalajara, Lleida, Madrid-Centre, Santiago de Compostela, Toledo, Valencia-PI, Valencia-PII and Valencia-QB, with median alcohol consumption in Bilbao between 1.5 (Valencia-PII) and 3 (Toledo) times higher than in the aforementioned cities. Also, statistically significant differences were observed between Palma de Mallorca and Toledo (consumption in Palma de Mallorca 2 times higher than in 
Toledo) and between Móstoles and Castellón (consumption in Móstoles 1.7 times higher than in Castellón) (Table 1 and S3).

At the regional level (Figure 1b, Table S4) differences of alcohol consumption were statistically significant ( $\mathrm{p}$-value $<0.05$, U Mann Whitney test) between Basque Country and all the other investigated regions, except Catalonia, and between Balearic Islands and the region of CastillaLa Mancha and Galicia (Table S4). The median consumption of alcohol in the Basque Country (19 $\mathrm{mL} /$ day/inhabitant) was between 1.5 and 2.2 times higher than the median consumption observed in Balearic Islands (12), Community of Madrid (11), Valencian Community (9.5), Castilla-La Mancha (8.7) and Galicia (8.5 mL/day/inhabitant). Balearic Islands presented a median figure of alcohol consumption 1.5 times higher than those obtained in Castilla-La Mancha and Galicia.

As for the city size, small cities, i.e., those with official census populations $<300,000$ inhabitants (Toledo, Guadalajara, Santiago de Compostela, Reus, Tarragona, Lleida, Castellón and Móstoles), showed significantly lower alcohol consumption rates per capita than large cities, i.e., those with official census population >300,000 (p-value < 0.05, U Mann Whitney).

\subsection{Weekly patterns}

Figure 2 shows the daily alcohol consumption expressed as $\mathrm{mL} / \mathrm{day} / \mathrm{inhabitant}$ or as the contribution of each day to the total weekly consumption observed in each population. The difference in the amount of alcohol consumed during the weekend (Saturday and Sunday) (median=15 mL/day/inhabitant) and during the weekdays (Monday to Friday) (median=9.0 $\mathrm{mL} /$ day/inhabitant) was found to be statistically significant ( $\mathrm{p}<0.05$, U Mann Whitney).

Figure S2 shows the weekly trends of alcohol consumption in the investigated populations. The strongest differences in alcohol consumption between weekdays and weekends were observed in Reus and Toledo (with average consumption figures 2.2 and 2.0 times higher, respectively, during the weekend than during weekdays), and the weakest in Madrid-North (where Monday is the day of highest consumption) and Tarragona (where, in fact, large variations in alcohol consumption were observed throughout the week (Figure S2)).

Figure 2 also shows a general high contribution of Mondays to total weekly alcohol consumption figures when compared with the other weekdays. According to Høiseth et al., EtS can remain in urine for several hours (between 25 and 48) depending on the dose of ethanol ingested (Høiseth et al., 2008), so, the high value of alcohol consumption estimated on Monday could be attributed to the presence of EtS in wastewater from its consumption during the weekend.

\subsection{Nationwide extrapolation}

The total daily alcohol load (Kg/day) that arrived at each WWTP was used to back-calculate alcohol consumption at national level. Data were extrapolated taking into account that the population covered by the study was about 6.0 million inhabitants $(12.8 \%$ of the Spanish population) and the total population of Spain in 2018 accounted for 46.7 million inhabitants (INE, 2018). The extrapolation resulted in an annual consumption of $4.8 \pm 1.1 \mathrm{~L}$ of pure ethanol 
per capita in Spain, which increases to $5.7 \pm 1.2 \mathrm{~L}$ or $5.9 \pm 1.3 \mathrm{~L}$ of pure ethanol when only population above 15 years (aged 15+) or adult population (aged 18+) is considered, respectively (Table S5). This value is in line with official data reported by the National Health Survey (INE) (Table S6) that reports an average weekly consumption of $13 \mathrm{~mL} /$ day/inhabitant (aged 15+) equivalent to an average annual consumption of $4.7 \mathrm{~L}$ of pure ethanol per capita (aged 15+), and also with official data published by the Spanish Ministry of Agriculture, Fishing and Food, which indicates a consumption of beer of $51.8 \mathrm{~L}$ per capita (+18) (MAPA, 2018), equivalent to $4.3 \mathrm{~L}$ of pure ethanol per capita (aged 18+) taking into account that alcohol consumption by type of alcoholic beverage is distributed as 54\% beer, $18 \%$ wine and $28 \%$ spirits and the alcohol content in each one is $4.5,12$ and 40\%, respectively (WHO, 2018). On the contrary, higher alcohol consumption rate (10 L of pure ethanol per capita (aged 15+)) was reported for Spain in the WHO report (WHO, 2018).

\section{Discussion}

In this study, alcohol consumption in different populations of Spain was estimated by means of WBE. The population investigated covers $12.8 \%$ of the total Spanish population and is distributed around 13 main cities and 7 different regions. Results showed spatial variations in alcohol consumption among specific populations and among regions. Although Tarragona, Bilbao and Móstoles were the cities with the highest average alcohol consumption figures, Bilbao was the only one where alcohol consumption was significantly different to several other populations (see Table S3 and Figure 1). Also, alcohol consumption in Palma de Mallorca and Móstoles was significantly higher than in Toledo and Castellón, respectively. WBE-derived alcohol consumption figures were compared with the latest data reported by the National Health Survey carried out by the Spanish Ministry of Health, Consumption and Social Welfare in collaboration with the National Institute of Statistics (INE) (INE, 2017) and with prevalence data reported in the Annual Report of the Spanish Observatory on Drugs and Drugs Addiction (OEDA, 2019). Since official data are only provided at the level of regions, the average alcohol consumption obtained in each investigated population was compared with consumption data reported for its corresponding region. Figure 3 compares WBE data and INE National Health Survey data. WBE-derived alcohol consumption figures in five of the investigated populations (Toledo, Lleida, Madrid-Centre, Castellón, and Valencia-QB) showed good correlation with INE official data at the region level, being the differences of consumption figures lower than $13 \%$, whereas a weaker correlation (differences of consumption between 22 and 30\%) was observed in 4 populations (Palma de Mallorca, Reus, Valencia-PI, and Valencia-PII). WBEderived data in the remaining populations (Bilbao, Guadalajara, Barcelona, Tarragona, MadridNorth, Móstoles, and Santiago de Compostela) showed larger differences with official INE data.

On the other hand, comparison of WBE-data with prevalence data of alcohol consumption reported for each region, showed poor correlation when all investigated populations were considered (see Figure S3). However, as shown in Figure 4, when the data from the 7 populations that did not correlate with official INE consumption figures (Bilbao, Guadalajara, 
Barcelona, Tarragona, Madrid-North, Móstoles, and Santiago de Compostela) were removed, a significant correlation was observed ( $\mathrm{r}^{2}$ "Lifetime prevalence": 0.4499, $\mathrm{p}$-value $<0.05 ; \mathrm{r}^{2}$ "Last year prevalence": 0.5407 , p-value < 0.05). WBE-data showed that the population belonging to the Basque Country presented a significantly higher consumption than populations belonging to the other regions (except Catalonia) and also that alcohol consumption in the Balearic Islands was significantly higher than in those belonging to Castilla-La Mancha and Galicia. These results are in agreement with prevalence data only in the case of Balearic Islands since according to prevalence data reported by the Annual Report (Figure S4), Balearic Islands shows higher prevalence of consumption than Castilla-La Mancha and Galicia. However, in the case of the Basque Country, prevalence of alcohol consumption, although above the Spanish average, is similar to that reported for the Valencian Community or Galicia (Figure S4).

The differences observed between WBE-derived alcohol consumption figures and established indicators could have different explanations. On the one hand, the populations sampled may not be representative of alcohol consumption in the whole region. As previously demonstrated, significant differences in alcohol consumption were observed between small and large populations (section 3.2). In addition, in some cases, only one municipality was sampled in a specific region (i.e., Balearic Islands and Galicia) which may not adjust to the alcohol consumption patterns of the whole region. This hypothesis is supported by the fact that within the same region, WBE-data derived from some populations correlated well with the INE survey data, whereas others did not (see Castilla-La Mancha, Catalonia and Community of Madrid in Figure 3). Thus, increasing the population sampled or sampling populations of different size within one region, could lead to a more representative picture of the habits of consumption of the whole region. Despite this, at the national level, the annual alcohol consumption rate obtained by means of WBE approach was comparable to that reported by the National Health Survey, which may indicate that the sampled population is quite representative of the whole country.

On the other hand, data reported by established methods may also not represent the actual consumption by the population since they are also affected by certain uncertainty. In fact, the two established indicators used to compare the WBE-derived estimates, provide different results, in the sense that the highest prevalence data are reported for Balearic Islands (see Figure S4), whereas the highest alcohol consumption rate was reported for Galicia in the INE National Health Survey (Figure 3).

As expected, the weekly consumption patterns in most populations showed an increase in alcohol consumption during the weekend. Saturday and Sunday were the days when alcohol consumption contributed the most to the total weekly consumption, with a median contribution of $20 \%$, while the remaining days of the week contributed between $11 \%$ (Tuesday) and $14 \%$ (Monday) (Figure 2b). Similar results were obtained in Australia, where each weekend day contributed with $20 \%$ to the weekly consumption rate, while the rest of the days of the week varied between $11 \%$ and 13\% (Lai et al., 2018). The increase in alcohol consumption during the weekend was also reported in an international study conducted in 11 different countries worldwide (Baz-Lomba et al., 2016), in Norway (Reid et al., 2011), Belgium (Boogaerts et al., 2016; van Wel et al., 2016), and in Spain, where previous studies, far less ambitious than the 
present study, were done in Barcelona (Mastroianni et al., 2017, 2014), Santiago de Compostela (Rodríguez-Álvarez et al., 2015, 2014) and Valencia (Andrés-Costa et al., 2016). The increase of alcohol consumption during the weekend was also reported by the INE National Health Survey for all regions investigated in the present study in terms of consumption rate (see Table S6) (INE, 2017), so again, good correlation was obtained between WBE approach and established indicators.

Unlike the Spanish National Health Survey, WBE-derived data show low correlation to those reported by the WHO. This fact was also observed in the nation-wide study carried out in Belgium (Boogaerts et al., 2016) in which the national alcohol consumption rate estimated by WBE approach was half than that reported by WHO. Such differences can be attributed to the fact that WHO data may not be appropriately represent the actual consumption of alcohol by the population. WHO data are derived from production, import, export and sale data, which in countries where there is not a strict control, like Spain, can lead to an overestimation of consumption, since alcohol can be stored and not consumed shortly after purchase. In this sense, it has been checked that in countries like Norway, where sales statistics are among the most accurate in the world, good correlation was obtained between WBE and WHO data (Reid et al., 2011). Also it is important to mention that, in this study, WBE data have been obtained from samples collected during only one week which may not be representative of alcohol consumption throughout the entire year. Increasing the sampling period, several times a year or during consecutive years, could be used to obtain temporal trends in alcohol consumption within one year and throughout the years.

Although, good correlation has been mostly obtained between WBE-derived data and those obtained with established indicators, the estimates of alcohol consumption by means of WBE have associated other uncertainties that should be taken into consideration. On the one hand, it has been shown that EtS is stable in wastewater (one week at room temperature and more than 1 month at $-20^{\circ} \mathrm{C}$ ) (Rodríguez-Álvarez et al., 2014), however, in sewage systems EtS could be degraded to some extent (Banks et al., 2018; Gao et al., 2018). This could lead to an underestimation of the real alcohol consumption, which could (partially) explain the lower consumption estimates obtained by means of WBE compared to those reported by the WHO. Although, as demonstrated in a recent study conducted in Australia, degradation can be corrected by applying a correction factor (Zheng et al., 2020). On the other hand, the excretion rate used to back calculate alcohol consumption was obtained from two studies in which only 10 men (Høiseth et al., 2008) and one man (Wurst et al., 2006) were investigated, respectively. Further studies involving more volunteers of different age, gender or race, or studying the excretion rate among Spanish population could help to obtain a more representative excretion rate which would increase the accuracy of back-calculations. Finally, other sources of uncertainty may come from the sampling (collection of a not representative sample), inaccurate measurement of the water volume entering the plant, and the calculation of the size of the population that contributes to the total EtS load measured in wastewater (Castiglioni et al., 2013). In the present study, the latter was assessed using different methods (census data, population connected to the WWTP, water quality parameters), following in each case the 
recommendations provided by the experts of the WWTP in order to obtain the value that best reflects the population served by each WWTP.

Regardless of the aforementioned limitations, the WBE approach appears as a promising, convenient tool for alcohol consumption assessment, which surely needs to be refined in the next few years. WBE is much useful to establish spatial and temporal variations in alcohol consumption in a fast, objective and inexpensive way, providing data in nearly real-time. WBE can complement in this way the information gained with the established methodologies which are also affected by some uncertainties. In this sense, the use of different indicators and sources of information would definitely improve the alcohol consumption estimates and hence, contribute to a better development and evaluation of health care management plans and policies.

\section{Conclusions}

The present work represents the first nation-wide study conducted in Spain to evaluate alcohol consumption through the application of the WBE approach, and is one of the first nation-wide assessments available worldwide. The study has covered 13 main cities (in some cases including surrounding towns) that represent $12.8 \%$ of the Spanish total population. The results show that WBE is a useful tool to define spatial and temporal variations in alcohol consumption in a fast, objective and inexpensive way, providing complementary data to the information gained with the established methodologies. The WBE-derived alcohol consumption data correlated well (within $\pm 15 \%$ ) with official data reported by conventional methods at the region level in 5 out of the 16 populations investigated ( $31 \%$ of the total population examined), and satisfactorily (within $\pm 30 \%$ ) in 9 of the populations studied (accounting for $56 \%$ of the scrutinized population). Also, extrapolation of WBE-derived alcohol consumption estimates to the national territory led to an annual consumption of alcohol in Spain comparable to that reported for Spain by the National Health survey, although, lower than that reported by the WHO. The comparison of WBE data with those obtained with established consumption indicators should be done with caution because both methodologies are subject to some uncertainties. Increasing the sampling period, the sampled population, and conducting further studies on alcohol metabolism to establish appropriate correction factors would help to reduce the main uncertainties associated with WBE and, therefore, to improve the accuracy of the consumption estimates.

\section{Acknowledgments}

This work has been supported by the Spanish State Research Agency (AEI) CTM2016-81935REDT and CTM2017-84763-C3-1-R, CTM2017-84763-C3-3-R and CTM2017-84763-C3-R2. E. López-García acknowledges the financial support of the Generalitat of Catalonia (expedient number SA-2018-780 and Consolidated Research Group 2017 SGR 01404-Water and Soil Quality Unit). We also acknowledge the Galician Council of Culture, Education and 
Universities (ED481D 2017/003 and EM2014/004). Several of these projects are cofounded by the European Regional Development Fund.

The authors would like to acknowledge all the city councils of the cities participating in this study for their collaboration as well as the operators of the WWTPs for their assistance in the collection of the wastewater samples, especially to: Cristian Mesa and Angela Vidal from Aigues de Barcelona (Barcelona WWTP), Jordi Palatsi from Aqualia (Lleida WWTP), Viaqua and Concello de Santiago de Compostela, the Public Entity of Wastewater Treatment (EPSAR) of the Generalitat Valenciana and especially to Fernando Llavador. Luis Aceiton, Enrique Albors, Angel Jiménez, Maria José Tarrega, Sonia Tristante and all the personal of the WWTPs (Aguas de Valencia, Spain), Sociedad de Fomento Agrícola Castellonense (FACSA, Castellon), and especially WWTP operators Santiago Querol and Sara Gargallo are acknowledged for providing wastewater samples from Castellón, as well as Subdirección General de Gestión del Agua, Ayuntamiento de Madrid, for allowing the collection of samples from Madrid centro.

\section{References}

Andrés-Costa, M.J., Escrivá, Ú., Andreu, V., Picó, Y., 2016. Estimation of alcohol consumption during "Fallas" festivity in the wastewater of Valencia city (Spain) using ethyl sulfate as a biomarker. Sci. Total Environ. 541, 616-622. https://doi.org/10.1016/j.scitotenv.2015.09.126

Banks, A.P.W., Lai, F.Y., Mueller, J.F., Jiang, G., Carter, S., Thai, P.K., 2018. Potential impact of the sewer system on the applicability of alcohol and tobacco biomarkers in wastewaterbased epidemiology. Drug Test. Anal. 10, 530-538. https://doi.org/10.1002/dta.2246

Baz-Lomba, J.A., Salvatore, S., Gracia-Lor, E., Bade, R., Castiglioni, S., Castrignanò, E., Causanilles, A., Hernandez, F., Kasprzyk-Hordern, B., Kinyua, J., McCall, A.-K., van Nuijs, A., Ort, C., Plósz, B.G., Ramin, P., Reid, M., Rousis, N.I., Ryu, Y., de Voogt, P., Bramness, J., Thomas, K., 2016. Comparison of pharmaceutical, illicit drug, alcohol, nicotine and caffeine levels in wastewater with sale, seizure and consumption data for 8 European cities. BMC Public Health 16, 1035. https://doi.org/10.1186/s12889-016-36865

Boogaerts, T., Covaci, A., Kinyua, J., Neels, H., Van Nuijs, A.L.N., 2016. Spatial and temporal trends in alcohol consumption in Belgian cities: A wastewater-based approach. Drug Alcohol Depend. 160, 170-176. https://doi.org/10.1016/j.drugalcdep.2016.01.002

Castiglioni, S., Bijlsma, L., Covaci, A., Emke, E., Hernández, F., Reid, M., Ort, C., Thomas, K. V., Van Nuijs, A.L.N., De Voogt, P., Zuccato, E., 2013. Evaluation of uncertainties associated with the determination of community drug use through the measurement of sewage drug biomarkers. Environ. Sci. Technol. 47, 1452-1460. https://doi.org/10.1021/es302722f

Chen, J., Venkatesan, A.K., Halden, R.U., 2019. Alcohol and nicotine consumption trends in three U.S. communities determined by wastewater-based epidemiology. Sci. Total Environ. 656, 174-183. https://doi.org/10.1016/j.scitotenv.2018.11.350

European Monitoring Centre for Drugs and Drug Addiction, 2016 (EMCDDA, 2016). 
http://www.emcdda.europa.eu/system/files/publications/2273/TDXD16022ENC_4.pdf (accessed 3.9.20).

Gao, J., Li, J., Jiang, G., Yuan, Z., Eaglesham, G., Covaci, A., Mueller, J.F., Thai, P.K., 2018. Stability of alcohol and tobacco consumption biomarkers in a real rising main sewer. Water Res. 138, 19-26. https://doi.org/10.1016/j.watres.2018.03.036

Gao, J., Zheng, Q., Lai, F.Y., Gartner, C., Du, P., Ren, Y., Li, X., Wang, D., Mueller, J.F., Thai, P.K., 2020. Using wastewater-based epidemiology to estimate consumption of alcohol and nicotine in major cities of China in 2014 and 2016. Environ. Int. 136, 105492. https://doi.org/10.1016/j.envint.2020.105492

Gatidou, G., Kinyua, J., van Nuijs, A.L.N., Gracia-Lor, E., Castiglioni, S., Covaci, A., Stasinakis, A.S., 2016. Drugs of abuse and alcohol consumption among different groups of population on the Greek Island of Lesvos through sewage-based epidemiology. Sci. Total Environ. 563-564, 633-640. https://doi.org/10.1016/j.scitotenv.2016.04.130

González-Mariño, I., Baz-Lomba, J.A., Alygizakis, N.A., Andrés-Costa, M.J., Bade, R., Barron, L.P., Been, F., Berset, J.-D., Bijlsma, L., Bodík, I., Brenner, A., Brock, A.L., Burgard, D.A., Castrignanò, E., Christophoridis, C.E., Covaci, A., de Voogt, P., Devault, D.A., Dias, M.J., Emke, E., Fatta-Kassinos, D., Fedorova, G., Fytianos, K., Gerber, C., Grabic, R., Grüner, S., Gunnar, T., Hapeshi, E., Heath, E., Helm, B., Hernández, F., Kankaanpaa, A., Karolak, S., Kasprzyk-Hordern, B., Krizman-Matasic, I., Lai, F.Y., Lechowicz, W., Lopes, A., López de Alda, M., López-García, E., Löve, A.S.C., Mastroianni, N., McEneff, G.L., Montes, R., Munro, K., Nefau, T., Oberacher, H., O’Brien, J.W., Olafsdottir, K., Picó, Y., Plósz, B.G., Polesel, F., Postigo, C., Quintana, J.B., Ramin, P., Reid, M.J., Rice, J., Rodil, R., Senta, I., Simões, S.M., Sremacki, M.M., Styszko, K., Terzic, S., Thomaidis, N.S., Thomas, K.V., Tscharke, B.J., van Nuijs, A.L.N., Yargeau, V., Zuccato, E., Castiglioni, S., Ort, C., 2019. Spatio-temporal assessment of illicit drug use at large scale: evidence from 7 years of international wastewater monitoring. Addiction. https://doi.org/10.1111/add.14767

Helander, A., Beck, O., 2005. Ethyl Sulfate: A Metabolite of Ethanol in Humans and a Potential Biomarker of Acute Alcohol Intake. J. Anal. Toxicol. 29, 270-274. https://doi.org/10.1093/jat/29.5.270

Høiseth, G., Bernard, J.P., Stephanson, N., Normann, P.T., Christophersen, A.S., Mørland, J., Helander, A., 2008. Comparison between the urinary alcohol markers EtG, EtS, and GTOL/5-HIAA in a controlled drinking experiment. Alcohol Alcohol. 43, 187-191. https://doi.org/10.1093/alcalc/agm175

Instituto Nacional de Estadística, 2017 (INE, 2017). Consumo medio diario de alcohol (en gramos de alcohol puro) según sexo y comunidad autónoma. Media y desviación tipica. Población de 15 y más años que consume bebidas alcohólicas uno o más días a la semana. https://www.ine.es/jaxi/Tabla.htm?path=/t15/p419/a2017/p03/10/\&file=03011.px\&L=0 (accessed 1.23.20).

Instituto Nacional de Estadística, 2018 (INE, 2018). Población residente por fecha, sexo y edad(9663). https://www.ine.es/jaxiT3/Datos.htm?t=9663 (accessed 3.3.20).

Lai, F.Y., Gartner, C., Hall, W., Carter, S., O’Brien, J., Tscharke, B.J., Been, F., Gerber, C., White, J., Thai, P., Bruno, R., Prichard, J., Kirkbride, K.P., Mueller, J.F., 2018. Measuring spatial and temporal trends of nicotine and alcohol consumption in Australia using 
wastewater-based epidemiology. https://doi.org/10.1111/add.14157

Ministerio de Agricultura, Pesca y Alimentación,

2018 (MAPA,

2018) https://cerveceros.org/uploads/5d1b32241b8be__Informe SocioeconomicoCerveza_2018.pdf (accessed 11.28.19).

Mastroianni, N., Lopez de Alda, M., Barcelo, D., 2014. Analysis of ethyl sulfate in raw wastewater for estimation of alcohol consumption and its correlation with drugs of abuse in the city of Barcelona. J. Chromatogr. A 1360, 93-99. https://doi.org/10.1016/j.chroma.2014.07.051

Mastroianni, N., López-García, E., Postigo, C., Barceló, D., López de Alda, M., 2017. Fiveyear monitoring of 19 illicit and legal substances of abuse at the inlet of a wastewater treatment plant in Barcelona (NE Spain) and estimation of drug consumption patterns and trends. Sci. Total Environ. https://doi.org/10.1016/J.SCITOTENV.2017.07.126

Nguyen, H.T., Thai, P.K., Kaserzon, S.L., O’Brien, J.W., Eaglesham, G., Mueller, J.F., 2018. Assessment of drugs and personal care products biomarkers in the influent and effluent of two wastewater treatment plants in Ho Chi Minh City, Vietnam. Sci. Total Environ. 631632, 469-475. https://doi.org/10.1016/j.scitotenv.2018.02.309

Observatorio Español de las Drogas y las Adicciones, 2019 (OEDA, 2019). INFORME 2019 Alcohol, tabaco y drogas ilegales en España.

Probst, C., Fleischmann, A., Gmel, G., Poznyak, V., Rekve, D., Riley, L., Rylett, M., Shield, K.D., Rehm, J., 2019. The global proportion and volume of unrecorded alcohol in 2015. J. Glob. Health 9. https://doi.org/10.7189/jogh.09.010421

Reid, M.J., Langford, K.H., Mørland, J., Thomas, K. V., 2011. Analysis and Interpretation of Specific Ethanol Metabolites, Ethyl Sulfate, and Ethyl Glucuronide in Sewage Effluent for the Quantitative Measurement of Regional Alcohol Consumption. Alcohol. Clin. Exp. Res. no-no. https://doi.org/10.1111/j.1530-0277.2011.01505.x

Rodríguez-Álvarez, T., Rodil, R., Cela, R., Quintana, J.B., 2014. Ion-pair reversed-phase liquid chromatography-quadrupole-time-of-flight and triple-quadrupole-mass spectrometry determination of ethyl sulfate in wastewater for alcohol consumption tracing. $\mathrm{J}$. Chromatogr. A 1328, 35-42. https://doi.org/10.1016/j.chroma.2013.12.076

Rodríguez-Álvarez, T., Racamonde, I., González-Mariño, I., Borsotti, A., Rodil, R., Rodríguez, I., Zuccato, E., Quintana, J.B., Castiglioni, S., 2015. Alcohol and cocaine co-consumption in two European cities assessed by wastewater analysis. Sci. Total Environ. 536, 91-98. https://doi.org/10.1016/j.scitotenv.2015.07.016

Ryu, Y., Barceló, D., Barron, L.P., Bijlsma, L., Castiglioni, S., de Voogt, P., Emke, E., Hernández, F., Lai, F.Y., Lopes, A., de Alda, M.L., Mastroianni, N., Munro, K., O’Brien, J., Ort, C., Plósz, B.G., Reid, M.J., Yargeau, V., Thomas, K. V., 2016. Comparative measurement and quantitative risk assessment of alcohol consumption through wastewater-based epidemiology: An international study in 20 cities. Sci. Total Environ. 565, 977-983. https://doi.org/10.1016/j.scitotenv.2016.04.138

Stockwell, T., Zhao, J., Greenfield, T., Li, J., Livingston, M., Meng, Y., 2016. Estimating 
under- and over-reporting of drinking in national surveys of alcohol consumption: identification of consistent biases across four English-speaking countries. Addiction 111, 1203-1213. https://doi.org/10.1111/add.13373

van Wel, J.H.P., Gracia-Lor, E., van Nuijs, A.L.N., Kinyua, J., Salvatore, S., Castiglioni, S., Bramness, J.G., Covaci, A., Van Hal, G., 2016. Investigation of agreement between wastewater-based epidemiology and survey data on alcohol and nicotine use in a community. Drug Alcohol Depend. 162, 170-175. https://doi.org/10.1016/j.drugalcdep.2016.03.002

World Health Organization, 2018 (WHO, 2018)https://apps.who.int/iris/bitstream/handle/10665/274603/9789241565639eng.pdf?ua=1 (accessed 11.15.19).

Wurst, F.M., Dresen, S., Allen, J.P., Wiesbeck, G., Graf, M., Weinmann, W., 2006. Ethyl sulphate: A direct ethanol metabolite reflecting recent alcohol consumption. Addiction 101, 204-211. https://doi.org/10.1111/j.1360-0443.2005.01245.x

Zheng, Q., Tscharke, B.J., Krapp, C., O’Brien, J.W., Mackie, R.S., Connor, J., Mueller, J.F., Thomas, K. V., Thai, P.K., 2020. New approach for the measurement of long-term alcohol consumption trends: Application of wastewater-based epidemiology in an Australian $\begin{array}{lllll}\text { regional city. Drug } & 207 .\end{array}$ https://doi.org/10.1016/j.drugalcdep.2019.107795 
Table 1. Frequency of detection of EtS $(\%)$, EtS concentration $(\mu \mathrm{g} / \mathrm{L})$, EtS load (mg/day/inhabitant) and alcohol consumption (mL/day/inhabitant) 624 in the investigated cities (expressed as average, median and range).

\begin{tabular}{|c|c|c|c|c|c|c|c|c|c|c|c|c|}
\hline & \multicolumn{4}{|c|}{ Concentration $(\mu \mathrm{g} / \mathrm{L})$} & \multicolumn{3}{|c|}{ EtS load (mg/day/inhabitant) } & \multicolumn{5}{|c|}{ Alcohol (mL/day/inhabitant) } \\
\hline & $\begin{array}{c}\text { Freq. } \\
\text { (\%) }\end{array}$ & Average & Median & Range & Average & Median & Range & Average & Median & Range & $\begin{array}{l}\text { Average } \\
\text { weekdays }\end{array}$ & $\begin{array}{l}\text { Average } \\
\text { weekend }\end{array}$ \\
\hline Palma I & 100 & 15 & 15 & $11-21$ & - & - & - & - & - & - & - & - \\
\hline Palma II & 100 & 18 & 16 & $14-26$ & - & - & - & - & - & - & - & - \\
\hline Palma de Mallorca ${ }^{a}$ & & - & - & - & 3492 & 3221 & 2581-4702 & 14 & 12 & $10-18$ & 12 & 17 \\
\hline Bilbao & 100 & 17 & 16 & $18-29$ & 5133 & 4867 & $3906-7632$ & 20 & 19 & $15-30$ & 19 & 23 \\
\hline Guadalajara & 100 & 9.3 & 7.8 & $6.5-15$ & 2857 & 2499 & 2051-4417 & 11 & 9.7 & $7.9-17$ & 9.0 & 16 \\
\hline Toledo & 100 & 11 & 9.1 & 7.8-19 & 1926 & 1555 & $1426-3007$ & 7.4 & 6.0 & $5.5-12$ & 5.8 & 11 \\
\hline Barcelona & 100 & 16 & 14 & $5.9-25$ & 3455 & 3021 & $2030-5352$ & 13 & 12 & $7.8-21$ & 11 & 20 \\
\hline Lleida & 100 & 7.4 & 6.9 & $5.6-10$ & 2208 & 1807 & $1663-3333$ & 8.5 & 7.0 & $6.4-13$ & 7.2 & 12 \\
\hline Reus & 100 & 21 & 13 & $12-39$ & 3081 & 2036 & $1814-5363$ & 12 & 7.9 & $7.0-21$ & 8.8 & 20 \\
\hline Tarragona & 100 & 43 & 50 & $11-74$ & 7091 & 8597 & $1935-11906$ & 27 & 33 & $7.5-46$ & 27 & 28 \\
\hline Madrid-Centre & 100 & 15 & 15 & $9.4-23$ & 2301 & 2175 & $1381-3431$ & 8.9 & 8.4 & $5.3-13$ & 7.6 & 12 \\
\hline Madrid-North & 100 & 18 & 17 & $9.4-26$ & 3375 & 3342 & $1719-5327$ & 13 & 13 & $6.6-21$ & 13 & 14 \\
\hline Móstoles & 100 & 31 & 28 & $18-50$ & 4430 & 4147 & $2592-7520$ & 17 & 16 & $10-29$ & 15 & 22 \\
\hline Santiago de Compostela & 100 & 2.9 & 2.7 & $1.4-4.4$ & 2178 & 2197 & $1173-3124$ & 8.4 & 8.5 & $4.5-12$ & 7.0 & 12 \\
\hline Castellón & 100 & 12 & 11 & $7.3-23$ & 2325 & 1964 & $1635-4101$ & 9.0 & 7.6 & $6.3-16$ & 7.4 & 13 \\
\hline Valencia-PI & 100 & 13 & 13 & $7.5-19$ & 2977 & 2829 & $1722-4364$ & 12 & 11 & $6.6-17$ & 9.6 & 16 \\
\hline Valencia-PII & 100 & 12 & 11 & $6.9-19$ & 2957 & 3282 & $2168-3483$ & 11 & 13 & $8.4-13$ & 11 & 13 \\
\hline Valencia-QB & 100 & 14 & 11 & $10-22$ & 2438 & 2339 & $1693-3770$ & 9.4 & 9.0 & $6.5-15$ & 8.0 & 13 \\
\hline
\end{tabular}



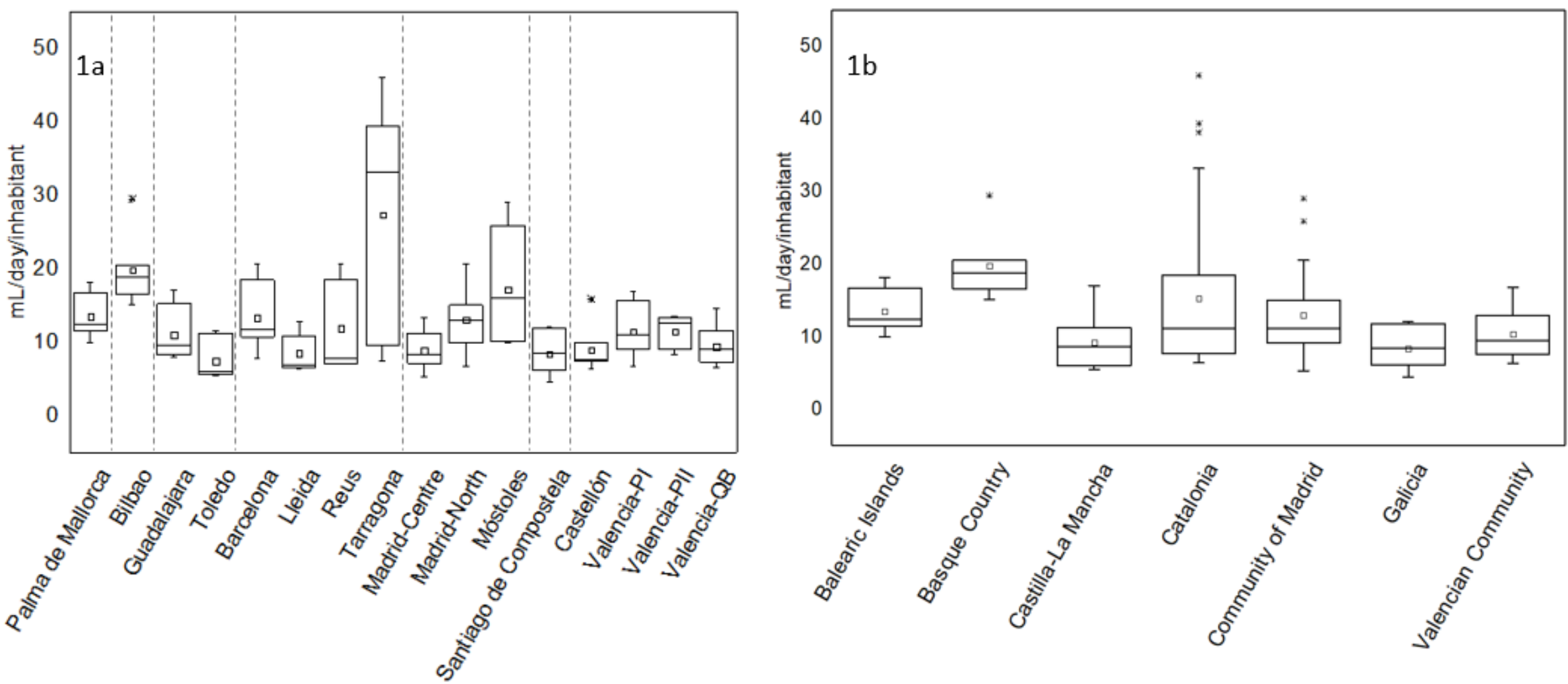

Figure 1. Distribution of alcohol consumption among investigated populations (Figure 1a) and among regions (Figure 1b). (In Figure 1a,

populations belonging to the same region are shown between vertical lines; * Outlier). 
630
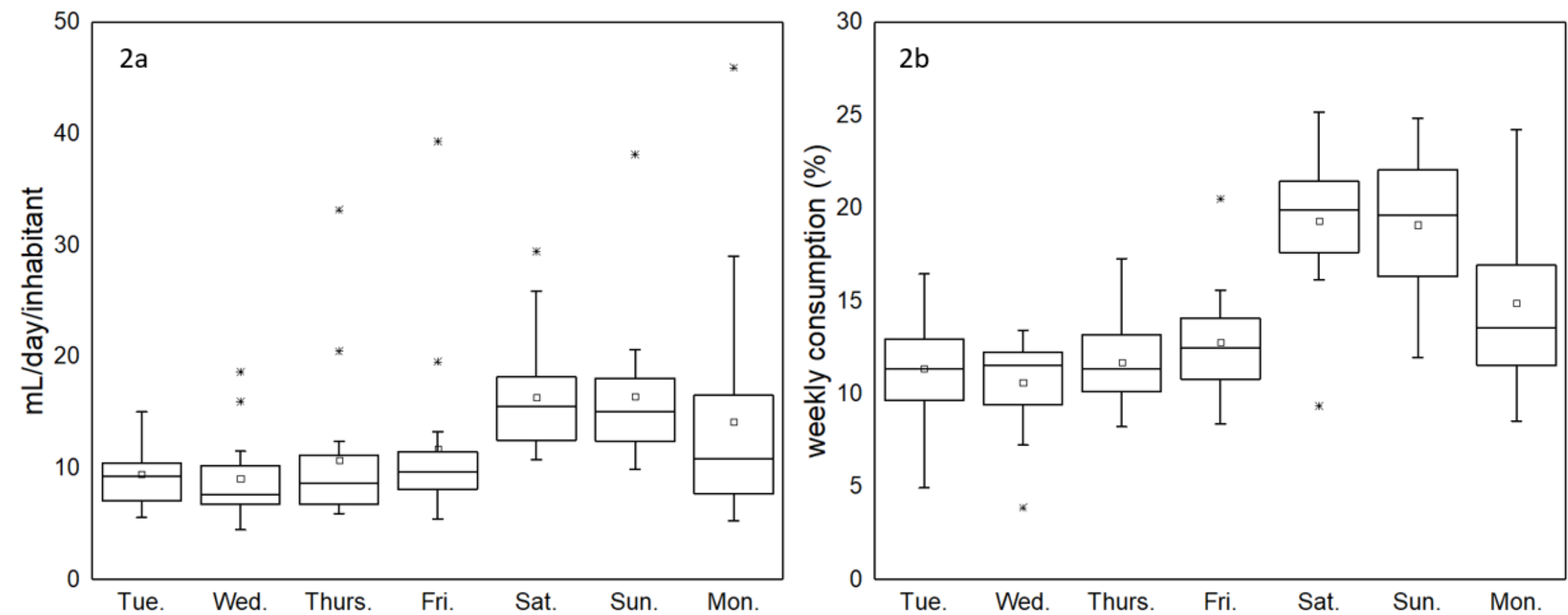

632 Figure 2. Distribution of alcohol consumption throughout the week expressed as mL/day/inhabitant (Figure 2a) and contribution of each day to 633 the total weekly consumption (\%) (Figure $2 b) .(*$ Outlier) 


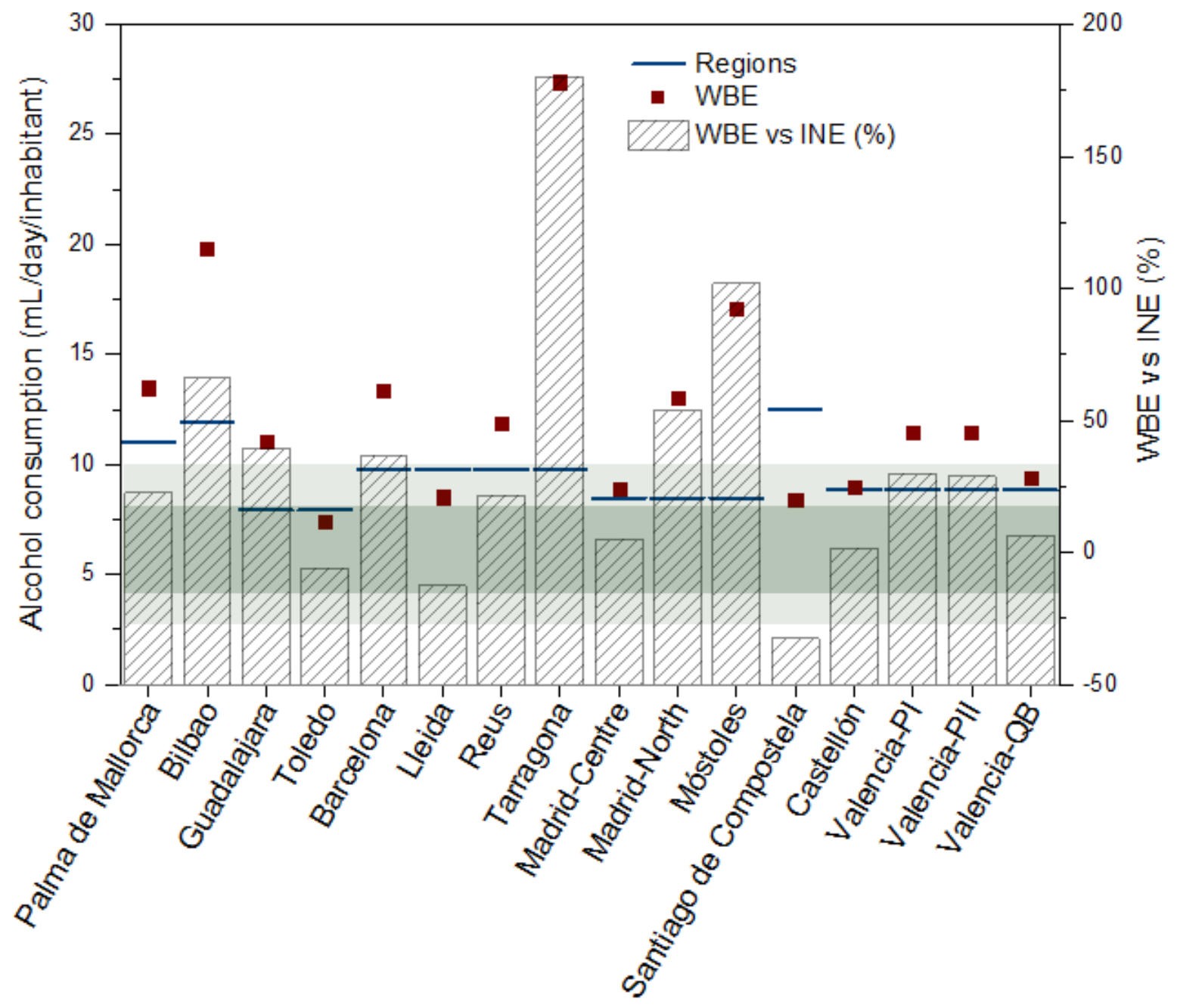

Figure 3. Alcohol consumption estimated in the investigated populations by means of WBE (red square), data reported for the corresponding region in the INE National Health Survey (blue line) and differences of consumption between WBE data and survey data (grated bars) (\%). (The bars within the dark green zone delimit consumption differences between both methodologies below $15 \%$ and those within the light green zone below 30\%)

641

642

643

644 


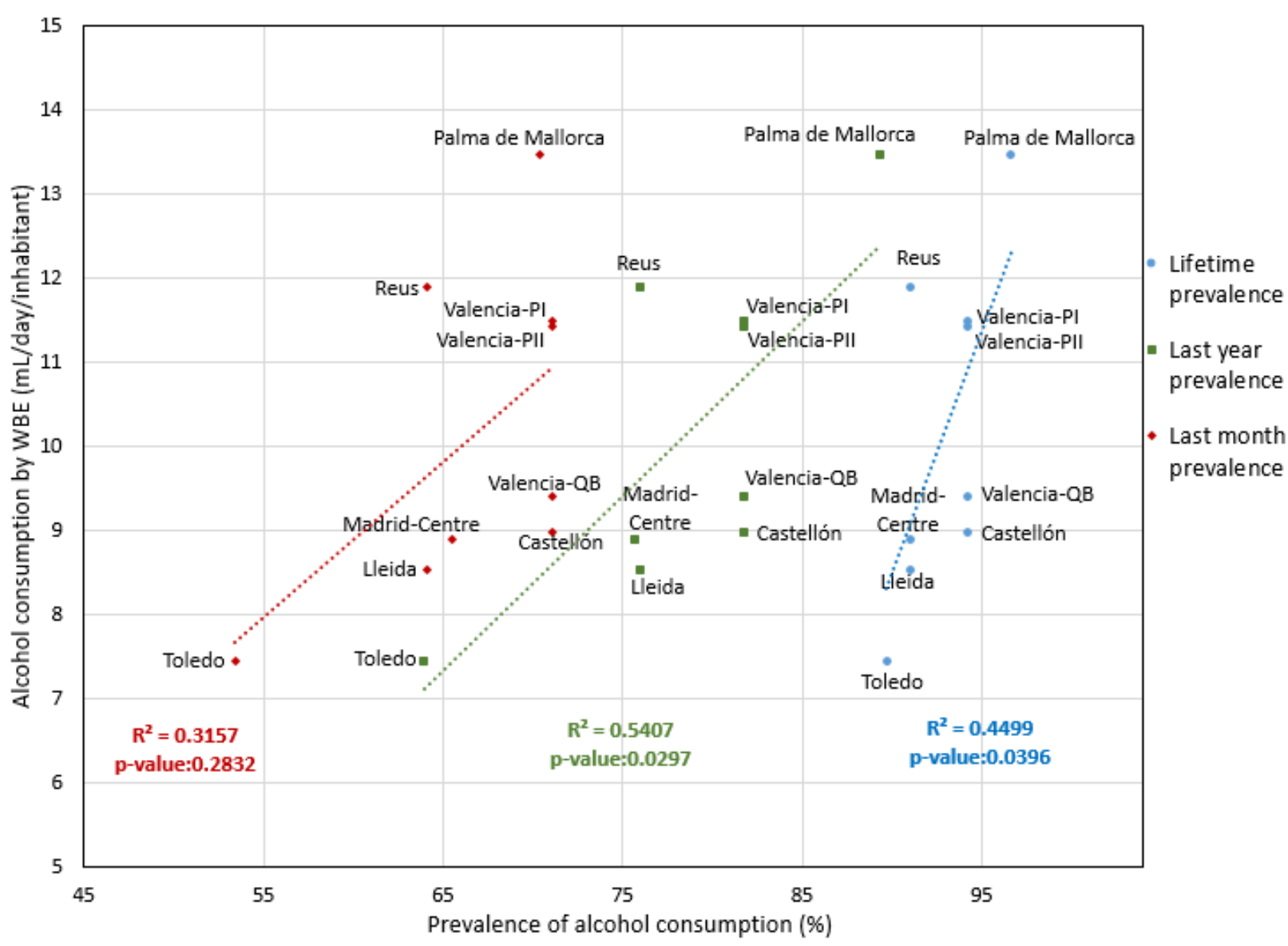

Figure 4. Correlation between average alcohol consumption estimated in each city by WBE 652 (mL/day/inhabitant) and prevalence data ("Lifetime prevalence", "Last year prevalence" and 653 "Last month prevalence") reported by its region in the Annual Report of the Spanish 654 Observatory on Drugs and Drugs Addiction 2019. (Data from Guadalajara, Barcelona, 655 Tarragona, Madrid-North, Móstoles, Santiago de Compostela and Bilbao were excluded; 656 Spearman correlation $\mathrm{p}$-value $<0.05$ were considered statistically significant). 